\title{
Das menschliche Maß
}

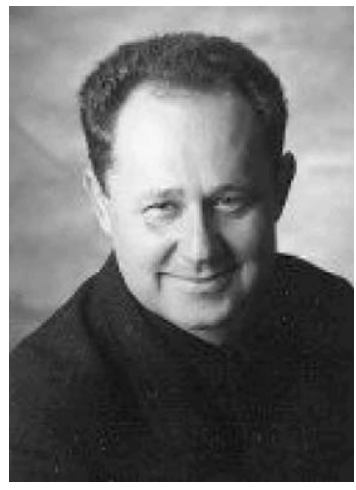

Prof. Dr. med. Manfred Wildner

\section{Bibliografie}

DOI http://dx.doi.org/ 10.1055/s-0033-1345181

Gesundheitswesen 2013;

75: $279-280$

(c) Georg Thieme Verlag KG

Stuttgart · New York

ISSN 0941-3790

Korrespondenzadresse

Prof. Dr. med. Manfred Wildner

Bayerisches Landesamt für Gesundheit und Lebensmittelsicherheit

Veterinärstraße 2

85764 Oberschleißheim

manfred.wildner@|gl.bayern.de
Der Mythos des Ikarus hat in der europäischen Kulturgeschichte vielfältige Spuren hinterlassen. Er wird gerne aufgegriffen, wenn menschlicher Übermut und menschliches Übermaß Gegenstand kritischer Betrachtungen sind. Was der Mythos erzählt? Daidalos und sein Sohn Ikarus wurden vom kretischen König Minos im Labyrinth des Minotauros, eines Mischwesens aus Stier und Mensch, gefangen gehalten. Der geschickte Daidalos, Erbauer des Labyrinths, hatte dem Theseus den Tipp gegeben, beim Betreten des Labyrinths einen Faden abzuwickeln. Damit konnte Theseus, nachdem er den Minotaurus getötet hatte, dieses wieder lebend verlassen. Der in Ungnade gefallene Daidalos war auch in eigener Sache findig: er konstruierte Flügel, für welche er Federn mit Wachs an einem Gestänge anbrachte. Selbst um die Risiken dieser Technik wissend, ermahnte er seinen Sohn Ikarus, nicht zu hoch und nicht zu tief zu fliegen, um einerseits der Hitze der Sonne und andererseits der Feuchtigkeit des Wassers zu entgehen. Nach anfänglich gelungener Flucht wurde Ikarus in der Freude über die neuen Möglichkeiten übermütig und kam in seinem Höhenflug der Sonne zu nahe - der darauf folgende Sturz des Ikarus ist bekanntes Kulturgut.

Ist der - nicht mein jugendliche - Übermut des Menschen Ursache seines Sturzes? Ist menschliches Unglück die Strafe der Götter für eine Missachtung des menschlichen Maßes? Offenbar stehen im Mittelpunkt der Geschichte der maßvolle Umgang mit Möglichkeiten und damit das dem Menschen gerechte Maß. Doch was ist das menschliche Maß? Ist es das Ideal des „Schneller, Weiter, Höher“, der Selbsttranszendenz des Menschen im Sinne des Perfekten? Dieses idealisierte Maß wäre eine mögliche Deutung. Ob dieses Maß auch gesundheitsverträglich ist? Die Lebenserwartung von olympischen Medaillengewinnern wie auch von Pulitzer- und Nobel-Preisträgern lässt hier durchaus Raum: Empirische Untersuchungen weisen eine im Vergleich mit den nicht ausgezeichneten Mitbewerbern deutlich bessere Lebenserwartungen der Preisträger aus [1,2]. Doch ist ein solches Maß auch problematisch: vor allem der Sieger bzw. die Siegerin profitiert [3]. Die Typologie eines Siegers und Preisträgers ist ja gerade durch die Unterlegenheit einer großen Zahl an Mitbewerbern charakterisiert. Zudem wären Fragen der Kausalität wie z.B. Selektionseffekte erst noch zu diskutieren. Für die Förderung der Bevölkerungsgesundheit taugt dieser Ansatz also nicht.

Oder ist das menschliche Maß womöglich das genaue Gegenteil? Ist unser Leben nur ein Wimpernschlag? Eine - insbesondere im Rückblick nur kurze Zeit zwischen Geburt und unvermeid- lichem Tod? Der Mensch ein „Mängelwesen“ (Arnold Gehlen), welches sich durch seine Kultur schützen muss [4]? Ist sein von Natur aus ungenügend gesichertes Dasein vielleicht immer auch ein Scheitern, ein Beladen sein mit Lasten und Krankheiten? Eindrückliche literarische Zeugnisse finden sich von der Hiobsgeschichte alter Zeiten bis zu den noch von Überlebenden bezeugten Schilderungen der Gräuel des Holocausts des 20. Jahrhunderts [5,6]. Eindrücklich ist auch die autobiografische Aufarbeitung medizinischer Extremsituationen wie dem Locked-in-Syndrom [7]. Doch ist nicht auch eine solche pessimistische Sicht wiederum ein Extrem?

Die Lebenswirklichkeiten des größten Anteils der Bevölkerung spielen sich, zumindest im geschützten Raum der wirtschaftlich entwickelten Nationen, im Dazwischen ab. Gesundheitswissenschaftliche Durchschnittswerte erzählen zum einen eine Geschichte von zumindest zeitweiligen Schmerzen und Erkrankungen. Sie erzählen gleichzeitig auch die Geschichte einer leistungsfähigen Medizin und einen in vielen Bevölkerungen im Durchschnitt guten Gesundheitszustand, bei geschichtlich noch nie erreichter Lebenserwartung mit anhaltend steigender Tendenz.

Gute Aussichten auf eine schöne neue Welt, erworben durch Vernunft, Wissensbasierung und soziale Verantwortung? Sicher nicht ohne eigene, neue Herausforderungen. Besondere Besorgnis erregt in westlich geprägten Gesellschaften die wachsende Last an psychischen Krankheiten. Die Ursachen dafür sind nicht klar. Die einfache Erklärung einer durch Enttabuisierung und wissenschaftlichen Fortschritt gestiegenen diagnostischen Wahrnehmung trägt nur ein Stück des Weges. Bei ADHS, „Burn-out“ oder Depressionen könnte auch die Morbiditätslast selbst zugenommen haben. Überfordern sich moderne Gesellschaften durch ihr rastloses Streben nach einem „Schneller, Weiter, Höher"? Sind diese psychischen Krankheiten Ausdruck der Erfahrung des stets Ungenügend-Seins angesichts überhöhter Selbst- und Fremdansprüche? Sind wir konstitutionell bzw. evolutionär nicht geschaffen für eine äußere Fülle, deren Kehrseite eine innere Leere ist? In moderner Nachfolge des findigen Daidalos werden immer neue Techniken und Apparate ersonnen, von PC und Laptop zu handheld devices wie Smartphone und Tablet: Die perfekte kommunikative Vernetzung einer mobilen Gesellschaft für den „flexiblen Menschen“ der Zukunft - unisex, versteht sich [8]. Unsere Lebens- und Arbeitswelten sind von Informationsströmen geprägt. Nicht mehr langsame Papierpost beherrscht unseren Arbeitstag, sondern flinke Elek- 
tronik: E-Mail, Telefonie, soziale Netze, in zunehmenden Maß mobil mit ständiger Verfügbarkeit. Mit dem möglichen Ergebnis des „erschöpften Selbst“: So der gleichnamige Titel des Buches von Alain Ehrenberg, in dem es darum geht, warum Depression die Krankheit unserer Zeit ist. Die Geschwindigkeit der Informationsverarbeitung wird dabei in Mega-, Giga- und inzwischen auch schon in Pentaflops pro Sekunde gemessen. Es ist zumindest vorstellbar, dass diese erfolgreichen Flops in der Informatik bei einer kritischen gesellschaftspolitischen Betrachtung als „Flops“ in einem anderen Sinn zu bewerten wären. Es sind die realen Freunde, die, auch empirisch nachweisbar, für das Lebensglück zählen, nicht virtuelle on-line-friends [9].

Neue Technologien treiben die gesellschaftlichen Entwicklungen. Das begleitend einzufordernde menschliche Maß wird kein statisches Maß wie der Pariser „Urmeter“ sein können. Menschliches Leben entfaltet sich dynamisch in einem sich mit entwickelndem, wesentlich von uns Menschen mit gestaltetem Umfeld. Damit finden wir uns in einer recht ähnlichen Situation wie Ikarus wieder, der von seinem nicht nur findigen, sondern auch recht klugen Vater zur Balance gemahnt werden musste. Zu einer Balance zwischen den Extremen der Möglichkeiten, welche als solche nicht unbedacht ausgeschöpft werden dürfen. Wie eine gelungene zivilisatorische Hülle aussehen könnte? Anpassungsfähig genug, um den vielen Ausprägungsformen menschlichen Lebens Geborgenheit und gleichzeitig Freiraum zu geben und, wo nötig, mit uns zu wachsen. Sowohl Idealnormen und Extreme wie auch die Durchschnittswerte und alle Schattierungen dazwischen umfassend. Offen für eine zeit- und ortsgebundene Diversität, dabei notwendigerweise auch auf universalen Werten aufbauend - vergleiche Hans Küngs Forderung nach einer „Weltethik“.

Was solche universalen Komponenten sein könnten? Vermutlich die gemeinsamen menschlichen Grunderfahrungen von Schmerz und Leid, von Hoffnung und Freude, die sich in der allgemeinen Menschenrechtserklärung und auch in den Verfassungen und Grundgesetzten der Länder mit Werten wie Menschenwürde, Freiheit und gesellschaftlicher Solidarität niedergeschlagen haben. Nicht zuletzt auch in den Sozialgesetzbüchern und den Gesetzen und Verordnungen, welche den Schutz, die Förderung und die Wiederherstellung der menschlichen Gesundheit zum Gegenstand haben. Dieses menschliche Maß ist nach aller Erfahrung auch nie abgeschlossen, sondern eine immer neu zu erarbeitende Balance. Bei aller Begeisterung auch für Höchstleistungen, für das Ungewöhnliche bis hin zur Ekstase als Möglichkeitsräume menschlichen Lebens sind unter gesundheitlichen Aspekten Exzesse meist weder hilfreich noch notwendig [10].

Wo sich solche gesunde Lebenswelten schaffen lassen? Hier sei an den Setting-Ansatz der Weltgesundheitsorganisation wie auch der Ottawa-Charta zur Gesundheitsförderung erinnert: Es geht um die Gestaltung der typischen Orte, an denen Menschen arbeiten, leben und lieben. Es handelt sich um die Familien, die Kindergärten und Schulen, die Arbeitsplätze, die Dörfer, Stadtviertel und Städte. Ansätze wie gesunde Schulen, gesunde Arbeitsplätze und gesunde Städte gehen in diese Richtung. Bisher wenig bedacht, jedoch im Bereich der gesundheitlichen Versorgung von zentraler Bedeutung, sind auch die Settings der Begegnungen zwischen den kranken oder von Krankheit bedrohten Menschen und ihren Helfern, den Ärztinnen und Ärzten und den vielfältigen therapeutischen Professionen: stationäre Einrichtungen, Ambulanzen und Praxen. Symbolisieren diese Orte, um im vorgestellten Bild zu bleiben, nicht die Grenzzonen zwischen dem balancierten Flug und dem gesundheitlichen Absturz? Sind die Menschen hier nicht gleichsam in der Phase, in der das
Wachs, welches unsere Federn zusammenhält, weich geworden ist und sie, besorgt und verstört, Hilfe suchen? Hier bieten sich besondere Chancen, neue Balancen zu finden. Einerseits um wieder zur Gesundheit ohne Krankheit und Leid zurückzufinden, andererseits auch, um vielleicht neue Formen von Gesundheit mit Krankheit einzuüben und zu akzeptieren.

Derartigen Fragen gehen die Beiträge in dieser Ausgabe wieder nach: Den Kosten und dem Nutzen eines klinischen Risikomanagements, Komplikationen im Krankenhaus und im Follow-up, den Folgen einer gerichtlichen Auseinandersetzung für betroffene (Zahn-)Ärzte, Burn-out bei Ärztinnen und Ärzten, objektivem Risiko und subjektiver Risikowahrnehmung am Beispiel Grippe, der Bestimmung von Pneumokokken-Impfquoten anhand von Routinedaten, der Frage der langfristigen gesundheitsbezogenen Lebensqualität nach stationärer Rehabilitation, der Einstellung zu klinischen Studien in Hausarztpraxen, der Finanzierung von Public Health in Europa und der Messung der Mitarbeiterzufriedenheit im Krankenhaus.

Vielleicht stellt sich bei diesen kulturhistorischen Rückgriffen auf alte Mythologien auch die Frage, ob solche Mahnungen überhaupt noch zeitgemäß sind. Haben wir es nicht doch geschafft, die Lüfte zu erobern, in noch größeren Höhen und mit noch Raum greifenderen Möglichkeiten, als dies der findige Daidalos für sich und seinen Sohn ersonnen hat? Haben wir uns nach einer Hansa der Meere nicht auch eine Hansa der Lüfte gebaut, welche uns auf technischen Kranichschwingen und mit Schallgeschwindigkeit nicht nur zu griechischen Inseln, sondern auch rund um den Globus tragen? Es sei jedem selber überlassen, wie weit er dieser hoffnungsfrohen Sichtweise zustimmen will. Was in jedem Fall zu ergänzen wäre, ist der Hinweis auf das, was diese Erfolgsgeschichte in der Luftfahrt erst möglich gemacht hat: neben Erfindergeist und Mut eine große Achtsamkeit und Sorgfalt. Diese ist im Flugverkehr exemplifiziert durch eine Herangehensweise, welche menschliche Fehler nicht ausblendet, sondern als Realitäten und sogar Chancen akzeptiert und Raum für eine angstfreie Fehlerkultur geschaffen hat, welche Abstürze wie die des Ikarus zunehmend zu vermeiden hilft. Dies ist ein Vorbild auch für das Gesundheitswesen (siehe z.B. http://www.ifpsbonn.de/). Der einsichtige, achtsame Umgang im Wissen um Potenziale wie auch Mängel unseres Menschseins und darauf aufbauend eine das menschliche Leben schützende und fördernde Kultur: vielleicht ist dies das dringend benötigte menschliche Maß.

\section{Literatur}

1 Clarke PM, Walter SJ, Hayen A et al. Survival of the fittest: retrospective cohort study of the longevity of Olympic medallists in the modern era. BMJ 2012; 345: e8308 doi:10.1136/bmj.e8308

2 Rablen MD, Oswald AJ. Mortality and immortality. University of Warwick, 2007.URL: http://www2.warwick.ac.uk/fac/soc/economics/ staff/academic/oswald/nobelsrablenos07.pdf Zugriff am 19.03.2012

3 Zwiers R, Zantvoord FWA, Engelaer FM et al. Mortality on former Olympic athletes: retrospective cohort analysis. BMJ 2012; 345: e7456 doi:10.1136/bmj.e7456

4 Gehlen A. Der Mensch. Seine Natur und seine Stellung in der Welt. Junker und Dünnhaupt. Berlin 1940

5 Wells LW. Ein Sohn Hiobs. Heyne Verlag; München: 1982

6 Pisar S. Das Blut der Hoffnung. Rowohlt Verlag; Berlin: 1979

7 Bauby JD. Schmetterling und Taucherglocke. DTV Verlag München: 1998

8 Sennett $R$. Der flexible Mensch. Die Kultur des neuen Kapitalismus Berlin-Verlag, Berlin 2002 [engl. The corrosion of character, New York: W.W. Norton, 1998]

9 Helliwell JF, Haifang $H$. Comparing the happiness effects of real and on-line friends. NBER Working Paper No. 18690, January 2013. URL: www.nber.org/papers/w18690, Zugriff am 19.03.2013

10 Bauman $A E$. Everyone could enjoy the "survival advantage" of elite athletes. BMJ 2012; 345: e8338 doi:10.1136/bmj.e8338 